\title{
Laser Interactions in Nanomaterials Synthesis
}

\author{
David B. Geohegan ${ }^{1,2}$, Alex A. Puretzky ${ }^{1,2}$, Chris Rouleau ${ }^{1,2}$, Jeremy Jackson ${ }^{1,2}$, \\ Gyula Eres ${ }^{2}$, Zuqin Liu ${ }^{1}$, David Styers-Barnett ${ }^{1}$, Hui Hu${ }^{2}$, Bin Zhao ${ }^{1}$, K. Xiao ${ }^{1}$, \\ Ilia Ivanov ${ }^{1,2}$, and Karren More ${ }^{2}$ \\ ${ }^{1}$ Center for Nanophase Materials Sciences and ${ }^{2}$ Materials Sciences and Technology \\ Divisions, Oak Ridge National Laboratory, Oak Ridge, Tennessee 37831 USA.
}

Laser interactions with materials have unique advantages to explore the rapid synthesis, processing, and in situ characterization of high quality and novel nanoparticles, nanotubes and nanowires. For example, laser vaporization of solids into background gases provides a wide range of processing conditions for the formation of nanomaterials by both catalyst-free and catalyst-assisted growth processes. Laser interactions with the growing nanomaterials provide remote in situ characterization of their size, structure, and composition with unprecedented temporal resolution. In this article, laser interactions involved in the synthesis of primarily carbon nanostructures are reviewed, including the catalyst-free synthesis of single-walled carbon nanohorns and quantum dots, to the catalyst-assisted growth of single and multi-walled carbon nanotubes.

\section{Introduction}

Laser vaporization of solid targets has long been a tool for the synthesis and discovery of clusters by mass spectrometry [1], resulting in the discovery of $\mathrm{C}_{60}$ and higher fullerenes in 1985 [2]. Two years later yttrium-barium-copper oxide high-temperature superconductors were discovered and commercial excimer lasers were found to congruently vaporize multicomponent targets to grow thin films of these materials,[3] fueling a resurgence of interest in pulsed laser deposition (PLD) for materials discovery, and a need to more fully understand the laser vaporization process.[4] In 1996, while trying to develop a catalystassisted process for mass production of fullerenes, laser vaporization of a multicomponent (carbon and metal catalyst) target into flowing argon gas at high temperatures $(1100 \mathrm{C})$ resulted in the synthesis single-wall carbon nanotubes, a major breakthrough in their production.[5] In 1998, this laser vaporization technique was generalized for the VLS-synthesis of semiconducting nanowires [6,7], further emphasizing the role of lasers in the exploration of new nanomaterials. These discoveries were highly instrumental in the development of an understanding of nanomaterials synthesis. In this article, we will outline some of the key processes governing the synthesis of nanomaterials by laser-driven interactions, with a special emphasis on carbon materials. 


\section{Laser Ablation and Plume Thermalization at Low Pressures}

The virtues of laser ablation for the pulsed laser deposition (PLD) of thin films primarily involve the rapid, stoichiometric removal and atomization of a solid, and the formation of an energetic beam of neutrals, ions, small molecules, and clusters [4]. The laser interaction with the solid usually forms a dense laser plasma $\left(T_{e} \sim 1-10 \mathrm{eV}\right)$ which expands and cools during a period of collisions near the target surface in which fast ions, slower neutrals, and even slower molecules and clusters emerge with a shifted, center-of-mass Maxwellian velocity distribution. Despite disparate masses, atoms in a multicomponent target often travel at nearly the same velocity when they emerge from this collisional "Knudsen layer", with atoms near the peak of the distribution typically moving at velocities $v \sim 1 \mathrm{~cm} / \mu \mathrm{s}$, corresponding to significant kinetic energies $(\sim 10-100 \mathrm{eV})$.

However, immediately following laser vaporization, oxidation and other chemical reactions can occur in the early portions of the plume expansion to form new molecules and clusters. In addition, since nanosecond or longer pulses are typically utilized, the laser may interact with the ejecta as they expand, resulting in photodissociation of clusters, photoionization of neutrals and other processes resulting in regional heating and secondary plume dynamics. An example of this is shown in Figure 1, where pyrolytic graphite is ablated by $\operatorname{ArF}(193 \mathrm{~nm})$ and $\mathrm{KrF}(248 \mathrm{~nm})$ lasers in vacuum. [8,9]

Stepwise increases in laser intensity results in the appearance of distinct regions

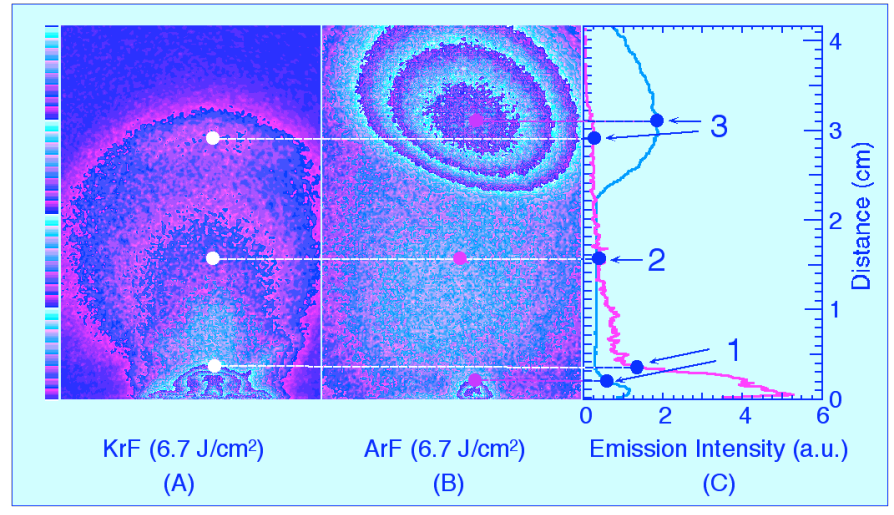

Fig. 2.1. ICCD images of visible plume emission from KrF-laser $(248 \mathrm{~nm})$ and ArFlaser $(193 \mathrm{~nm})$ ablated pyrolytic graphite in vacuum, taken $\Delta \mathrm{t}=1.0 \mu \mathrm{s}$ following ablation. Three regions of plume emission are observed, corresponding to (1) $C_{2}$ and $\mathrm{C}_{3}$, (2) C, and (3) $\mathrm{C}^{+}$. (Reproduced with permission from [8]). 
of plasma luminescence: first, from excited primary ejecta $\mathrm{C}_{3}$ and $\mathrm{C}_{2}$; second, from atomic carbon resulting from photodissociation of $\mathrm{C}_{2}$; and third, a fast ball of $\mathrm{C}^{+}$ions resulting from two-photon, resonant ionization of atomic $\mathrm{C}$ (Reproduced with permission from [10]). The interplume dynamics, which result in the selective acceleration of the $\mathrm{C}$ and $\mathrm{C}^{+}$, are observed to retard the expansion of the slower $\mathrm{C}_{2}$ and $\mathrm{C}_{3}$, inducing additional collisions and more clustering, and redeposition of these materials on the target surface. Thus, the choice of laser wavelength can influence the composition, kinetic energies, and trajectories of the initial ejecta from the target.
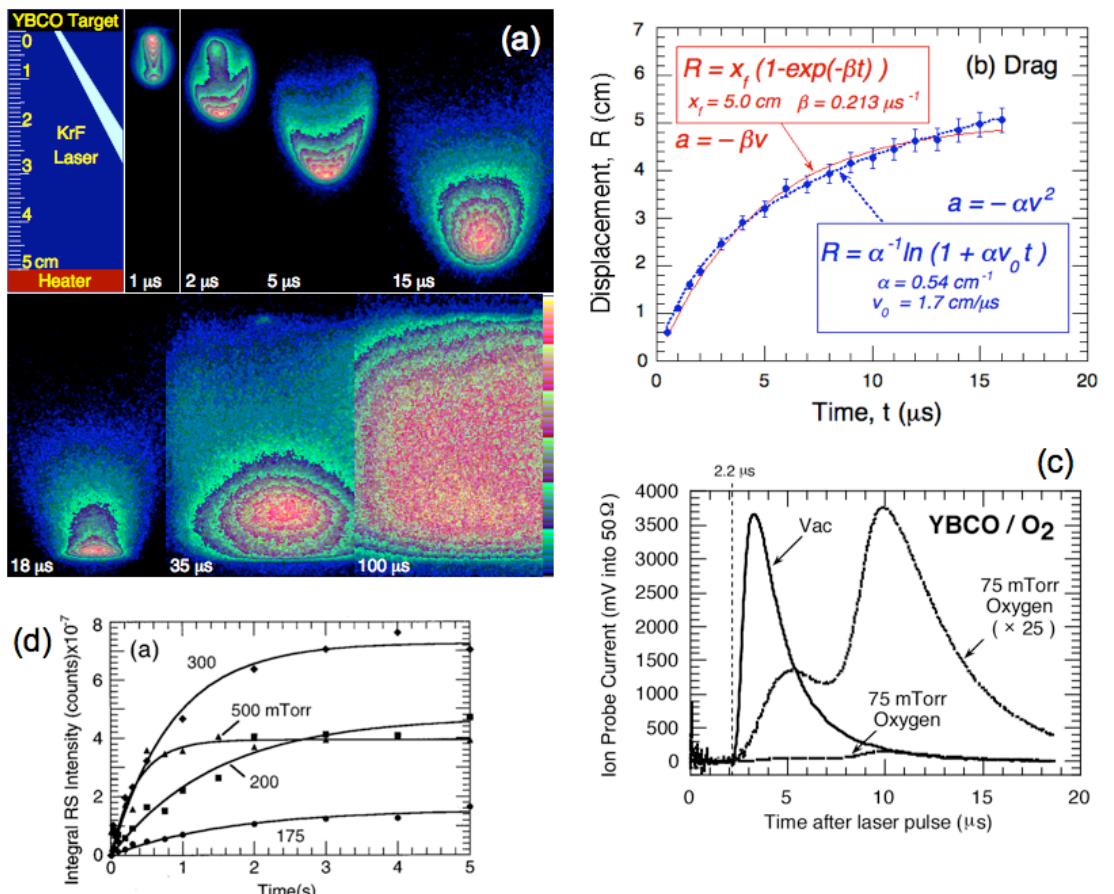

Fig. 2.2. (a) Side-on, false-color ICCD images of visible plume emission from YBCO ablated in $200 \mathrm{mTorr}$ oxygen at the indicated times. Although initially moving at leading edge velocities of $1 \mathrm{~cm} / \mathrm{us}$, the plume arrives at a heater surface $5 \mathrm{~cm}$ away at $\Delta \mathrm{t}$ $=15 \mu \mathrm{s}$. The plume does not entirely deposit, but rebounds to fill the region between the heater and target. [10] (b) The propagation of the leading edge of the plume is adequately represented by phenomenologic drag models. (c) However, ion probe flux measurements reveal a "splitting" of the plume at certain distances and pressures which has only been adequately explained by an elastic collision model. [10]. (d) Integrated intensities from Rayleigh scattering images of the region between the target and heater show the time dependences of nanoparticle growth at pressures typically used for PLD. [Adapted from Refs. 8,9,11] 
The addition of a low-pressure background gas results in collisions which slow the plume and confine it, often with the inadvertent formation of nanoparticles. Figure 2.2(a) shows a sequence of images of the plume resulting from YBCO ablation into 200 mTorr oxygen. Collisions between the plume atoms and ions with the background gas leads to bright recombination-fed fluorescence. Although this bright "shock front" progression can be adequately represented by shock and drag models, [4] two components of the plume coexist for a given range of distances for a particular background pressure, as revealed by ion flux measurements as in Figure 2.2(c). This "plume splitting" has been analyzed and modeled to result from elastic collisions which scatter and delay the plume atoms.[12,13] The two peaks roughly correspond to a fast distribution of material, exponentially-decaying with distance or pressure, of original plume material which has undergone few if any collisions - and a slowed peak which has undergone one or more collisions. After all the plume atoms have undergone several collisions, they form a slowed, propagating front of material which collides with a cold heater surface in Figure 2.2(a)(lower panel). A large fraction of the material does not stick to the heater surface, and slowly rebounds. During the next several seconds (Fig. 2.2(d)), laser-induced fluorescence imaging and Rayleigh scattering imaging (not shown) reveal that oxide clusters and nanoparticles slowly grow from this residual material for pressures above 175 mTorr under typical experimental conditions used for PLD film growth.[11] Interestingly, the imaging of Rayleigh scattered light from a time-delayed 308$\mathrm{nm}$ laser sheet revealed that this process is highly quenched by the application of a small temperature gradient, which flushes the nanoparticles from the region as they begin to form.[11]

\section{Synthesis of Nanoparticles by Laser Vaporization}

Novel new nanomaterials can be formed by laser vaporization into higher pressure background gases. $[14,15]$ The process can be modeled by isentropic expansion of a gas into vacuum, [16] however the actual dynamics are of interest in order to control the synthesis process. Figure 2.3 shows the plume expansion following laser vaporization of $\mathrm{Si}$ into 10 Torr He for the formation of brightly photoluminescent $\mathrm{SiO}_{\mathrm{x}}$ nanoparticles. For the first $400 \mu \mathrm{s}$, the plasma emission can be directly imaged however for longer times, a second, time-delayed $(308 \mathrm{~nm})$ laser is used to induce luminescence from the plume. In this case, for times $>200 \mu$ s the photoluminescence from small clusters and nanoparticles formed in the plume is used to reveal their position and dynamics. [17]

As the images show, a very bright region of photoluminescent clusters is formed behind the leading edge of the plume. These clusters were too small, however, to scatter light sufficiently for RS imaging. The nanoparticles grow and 

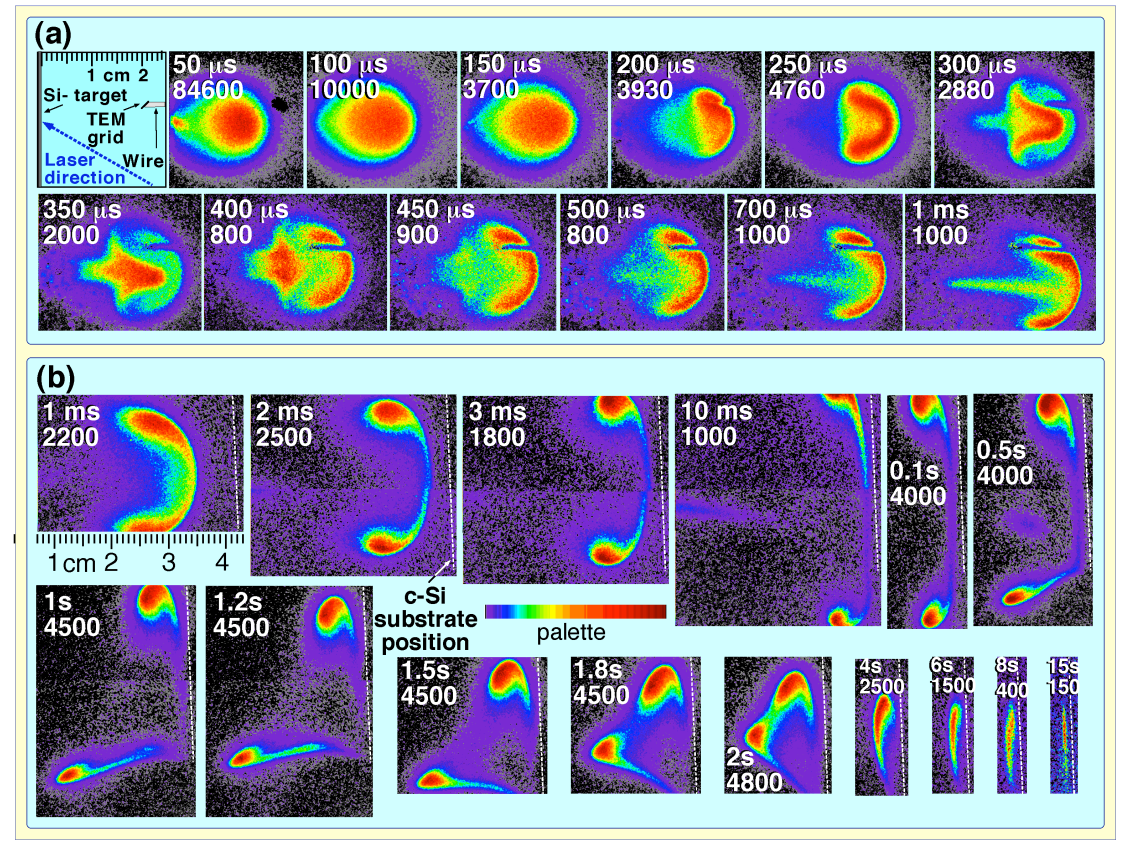

Fig. 2.3. (a) ICCD images of plasma luminescence $(\Delta \mathrm{t}<400 \mu \mathrm{s})$ plus photoluminescence $(\Delta t>200 \mu \mathrm{s})$ from nanoparticles produced by silicon ablation into 10 Torr He ( $3 \mu$ s exposures) at the indicated times and peak image intensities. (b) PL images utilizing a sheet of 308-nm laser light at later times show a slice through a swirling smoke ring of nanoparticles, and the nanoparticles encountering a roomtemperature silicon wafer (at the dashed line position). The movement of the lower portion of the nanoparticle cloud is due to a very weak gas flow in the chamber caused by the gas introduction. [17]

consolidate on the leading edge of the plume within $1 \mathrm{~ms}$, and the swirling, forward-moving vortex dynamic segregate the particles within a smoke ring which continues forward to encounter a stationary $\mathrm{Si}$ wafer at room temperature. The nanoparticles do not stick, but remain there for several seconds until they agglomerate, at which point photoluminescence is quenched.

These dynamics are quite unlike the expansion of ablated Si into background Argon (not shown). The high relative atomic mass of Ar vs. Si (39 vs. 14) induces significant slowing of the plume compared to the $\mathrm{Si} / \mathrm{He}$ case (4 vs. 14). Just 1 Torr of Ar produces a stopped and stationary cloud of nanoparticles (as revealed by RS imaging) without the turbulent motion needed to draw in oxygen required for oxidation into SiOx. Thus, without intentional flow of Ar to 
introduce trace impurities of oxygen, no PL is observed. Thus, the choice of background gas can significantly affect the propagation of the plume and its chemistry.

\section{Self Assembly of Carbon Fullerenes and Nanohorns}

Carbon fullerenes were discovered in 1985 by laser ablation of carbon into a high pressure background gas within a specially constructed, windowed pulsed nozzle source. [2] Soon after, production of fullerenes by laser vaporization of graphite targets within a hot tube furnace was used to scale the production of fullerenes to laboratory scale, and soon after electric arc vaporization was utilized for scaled production. [18] Theoretical modeling of the synthesis process has shown that high temperatures of $\sim 3000 \mathrm{~K}$ are required to induce the curvature necessary for the formation of fullerenes and other curved carbon nanostructures. Synthesis temperatures of $\sim 1000-2000 \mathrm{~K}$ produce flat carbon chain structures and sheets. Yet fullerenes and other larger nanostructures can

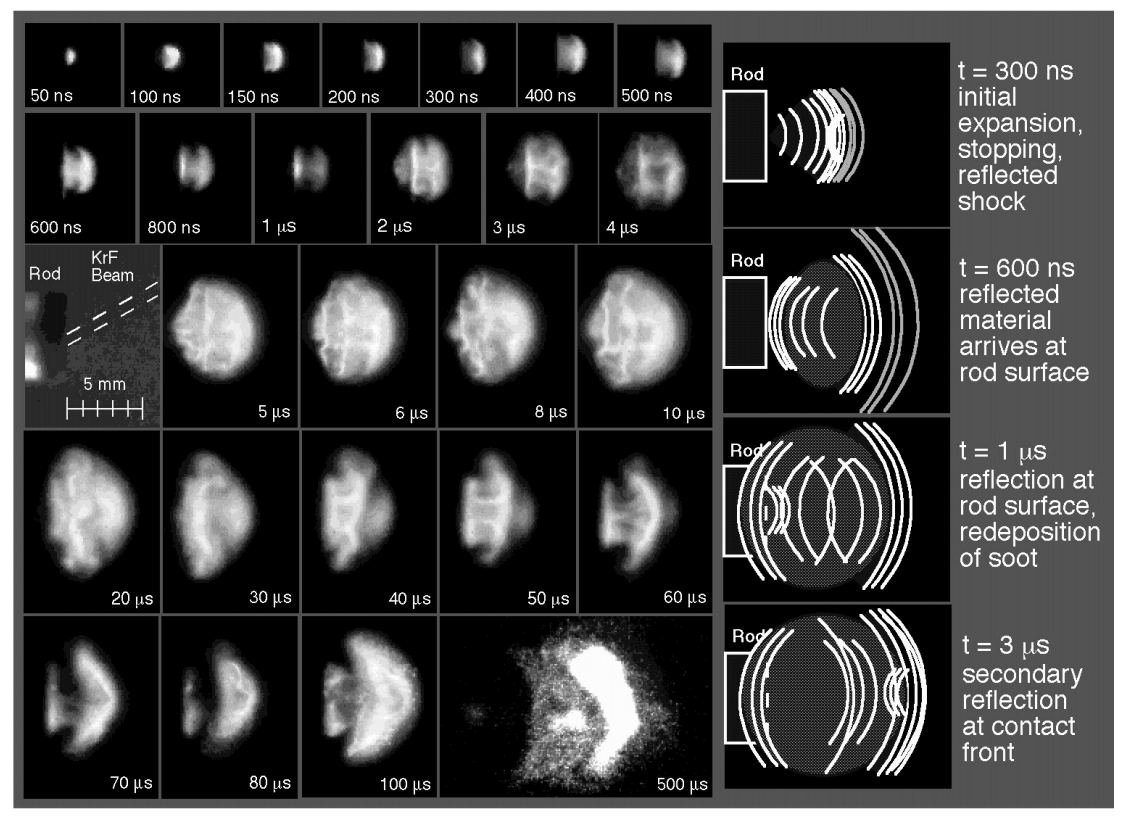

Fig. 2.4. (a) ICCD images of the interplume shock dynamics resulting from lase vaporization of $\mathrm{C}$ into 300 Torr $\mathrm{Ar}$ at room temperature for the formation of fullerenes The small quantity of ablated $\mathrm{C}$ is quickly (300 ns) stopped, and a reflected shock drive: material back toward the target. Reflected shocks continue, the plume expanding it oscillations, until a final push occurs in a mushroom cloud expansion where glowin clusters can be observed (at $500 \mu \mathrm{s}$ ). [19] 
be produced by laser vaporization into room-temperature background ambients.

To understand the timescales, temperatures, and dynamics involved in fullerene production time-resolved imaging and spectroscopy of the laser vaporization of carbon into room temperature 300 Torr Ar gas was performed (Figure 2.4). The images show a confined plume with a series of highly reproducible shock waves which correspond to regions of plume expansion and cooling. The initial expansion of high-density $\mathrm{C}$ atoms and ions is rapidly stopped (300ns) and a backward-propagating rarefaction wave is formed. This wave arrives at and reflects from the target surface from $\Delta \mathrm{t}=0.6-1.0 \mu \mathrm{s}$, and the plume is observed to oscillate and expand in stages as material oscillates between the contact front with the ambient gas and the target surface. During the process, material deposits on the target however no fullerenes are found there. The growth of the fullerenes occurs over extended times, during the final expansion of the plume for $\mathrm{t}>30 \mu \mathrm{s}$ after ablation. During this time, the plume cools from $\sim 3000 \mathrm{~K}$ to $\sim 1000 \mathrm{~K}$ as recorded by blackbody emission from hot clusters and particulates in the plume (as in $500 \mu$ s image in Fig. 2.4). Experimentally, the choice of background gas and pressure is found to govern the extent of plume confinement and the rate of cooling within the volume which serves as the substrateless microreactor where nanoparticle growth takes place. [19]

In 1999, much larger carbon nanostructures - single-wall carbon nanohorns (SWNHs) - were reported by a similar laser vaporization process, however at much higher laser power. [20] SWNHs are tubular shaped single-wall carbon nanostructures (like single-wall carbon nanotubes, SWNTs) however they are produced without catalysts. The synthesis process was not understood, however similar multiwalled tubular structures were formed in 1994 when "fullerene soot" from an arc reactor was annealed at high temperatures ex situ, indicating that in addition to completed fullerenes, incomplete carbon structures had been formed and were capable of further assembly. [21] The ablation of $\mathrm{C}$ targets into room temperature, atmospheric pressure background gases of $\mathrm{He}$ and $\mathrm{Ar}$ were found to form different flower-shaped aggregates of the nanohorns, including "dahlia-like" and "bud-like" nanohorns. [22]

Recently, we applied tunable laser pulses to investigate the timescales and dynamics of SWNH growth. $[23,24]$ By varying both the energy and the pulse width of a high-power (600-W average power) laser, different ablation regimes could be explored. To explore carbon nanostructures formed under long, continuous heating and ablation, the laser pulse width was adjusted to multimillisecond lengths and high energies (up to $100 \mathrm{~J} /$ pulse) were used. To explore nanostructures formed under shorter plume lifetimes, sub-millisecond pulses and low ( 1-5 J/pulse) laser energies were used. The temperature of the target surface was recorded by fast optical pyrometery during laser irradiation, and 


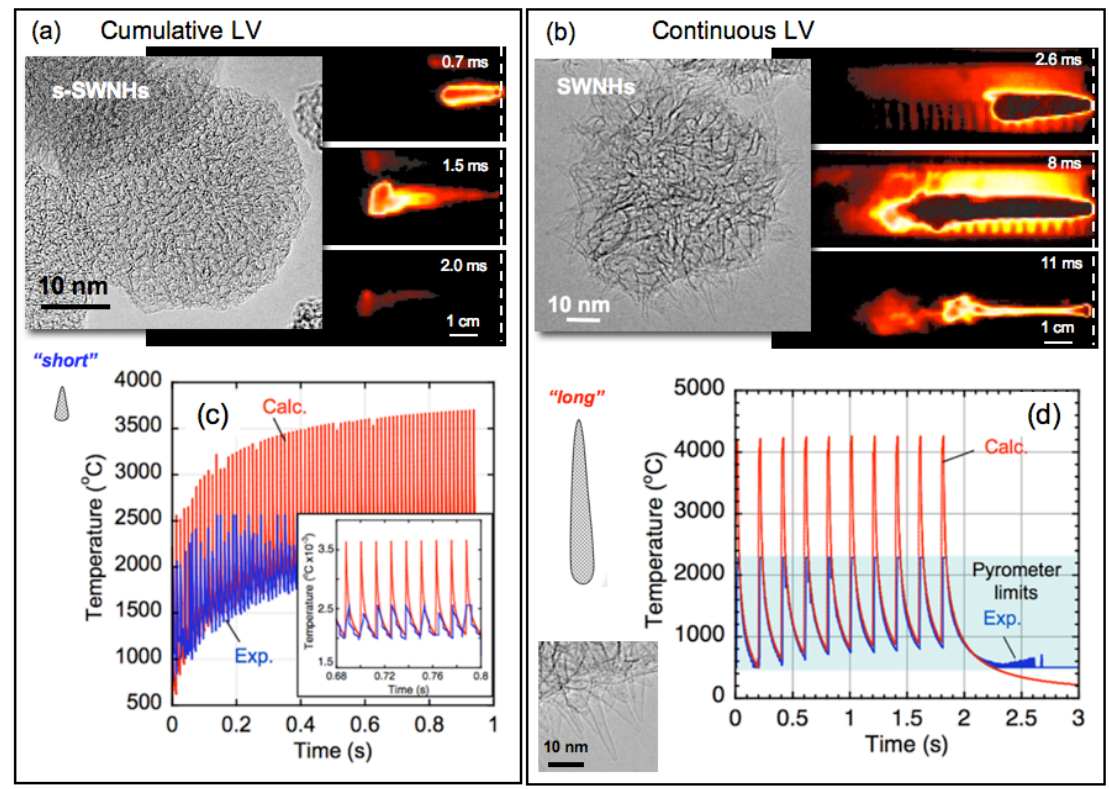

Fig. 2.5. (a), (b) Selected frames from high-speed (50,000 fps) video images recorded in situ from within a $1150^{\circ} \mathrm{C}$ tube furnace during high-power laser vaporization of $\mathrm{C}$ targets using (a) cumulative ablation (from $1 \mathrm{~ms}, 9 \mathrm{~J}$ laser pulses, at $50 \mathrm{~Hz}$ ) and (b) continuous ablation $(10 \mathrm{~ms}, 90 \mathrm{~J}$ laser pulses, $5 \mathrm{~Hz}$ ). Variation of the laser pulse widths and energies can be used to adjust the times and temperatures available for single-wall carbon nanotube and nanohorn growth. HRTEM images show representative materials collected outside the furnace following the synthesis events illustrated by the timeresolved image sequences. (c) and (d) illustrate in situ pyrometry of the target surface and calculated temperature profiles from a 3D heat transfer simulation of the target heating. Parameters are (c) $(20 \mathrm{~ms}$ pulses, $100 \mathrm{~J} /$ pulse, at $5 \mathrm{~Hz})$ and (d) short pulses $(0.5 \mathrm{~ms}$ pulses, $5 \mathrm{~J} /$ pulse at $80 \mathrm{~Hz})$. The highlighted horizontal band in (d) shows the pyrometer limits. After Refs. $[23,24]$

compared to a three-dimensional finite-element model simulation that included heating with a laser beam, heat losses due to heat conduction, target evaporation, black body radiation, and cooling by the surrounding buffer gas. The results are summarized in Figure 2.5. Cumulative laser vaporization with $1 \mathrm{~J}$ pulses was found to require $\sim 10$ laser pulses before the surface temperature was sufficient $(3750 \mathrm{C})$ to vaporize $C$, however once achieved a steady ablation rate of $\sim 6 \mathrm{~g} / \mathrm{h}$ was found to be very comparable to that using high-energy individual pulses for the same $\sim 500 \mathrm{~W}$ average laser power. On the other hand, individual high energy $(\sim 100 \mathrm{~J})$ pulses of 
10-20 ms duration were sufficient to rapidly heat the target to $4200 \mathrm{C}$, and maintain vaporization in a continuous ablation mode. High speed videography was used to record the heating and cooling times of the plume for comparison with the quite different nanohorn structures obtained in the different modes. As indicated in Figure 2.5 (a) and (b), high resolution TEM images show a variation in both the size of the individual nanohorn subunit, as well as the size of the aggregate structures which are formed. The length of nanohorn was found to correlate well with the time spent within the high temperature growth zone, with the length increasing at a rate of $\sim 1 \mathrm{~nm} / \mathrm{ms}$ of available growth time. This rate is highly comparable to the $\sim$ $1-5 \mathrm{~cm} / \mu \mathrm{s}$ rates found for catalyst-assisted SWNT growth, indicating that $\mathrm{C}$ can self-assemble into nanostructures at rates comparable to those using catalyst assistance.[24]

\section{Catalyst-Assisted Synthesis of Single-Wall Carbon Nanotubes}

Laser vaporization of carbon targets containing $~ 1-2$ at.\% metal catalyst powders (e.g. Ni and $\mathrm{Co}$ ) is a very effective technique to produce exclusively SWNTs at $\sim 1200^{\circ} \mathrm{C}$ in flowing Ar.[25] As summarized in Figure 2.6, in situ imaging and spectroscopy studies of the ns-laser vaporization process revealed that (a) both carbon and metal are prinicpally in the form of atoms and molecules $\left(\mathrm{C}, \mathrm{C}_{2}, \mathrm{C}_{3}, \mathrm{Ni}\right.$, Co) during the first $100 \mu \mathrm{s}$, when the plume of ejecta are within $\sim 1 \mathrm{~cm}$ of the target, (b) that carbon forms clusters within $1 \mathrm{~ms}$ after laser vaporization, as the hot plasma cools, and that (c) $\mathrm{Ni}$ and Co form clusters later in time $(1 \mathrm{~ms}<\mathrm{t}<2 \mathrm{~ms})$ after laser ablation. $[10,26]$ Through stop-growth experiments, where the plume was ejected from the hot oven after different growth periods (as revealed by imaging the plume via Rayleigh scattering shown in Fig. 2.5(e)), it was learned that only short SWNT "seeds" or nuclei had formed after $15-20 \mathrm{~ms}$ of growth time. By adjustment of this time, a growth rate in the range of $1-5$ microns/second could be inferred for SWNT growth by laser vaporization.[27]

It was concluded that one of the main conditions to achieve a high yield of SWNTs was confinement of the ejected material inside the propagating laser plume and that the main mechanism of this confinement was formation of a vortex ring. We recently showed that the confined volume could be significantly reduced if cumulative ablation using a sequence of pulses with a relatively low peak power (described above) was used to ablate the target instead of individual ns-laser pulses with high peak powers. The detailed study of this laser ablation regime revealed that preheating of the target with approximately ten laser pulses is required to achieve stationary ablation. Weight analysis of the target and HRTEM of the products revealed that, averaged over many pulses the same ablation rates were achieved for the same input total energy between single-shot and multi-shot ablation, but higher conversion efficiencies of carbon to SWNTs were obtained when the ejected material was confined in a smaller volume. [23] Therefore, this 


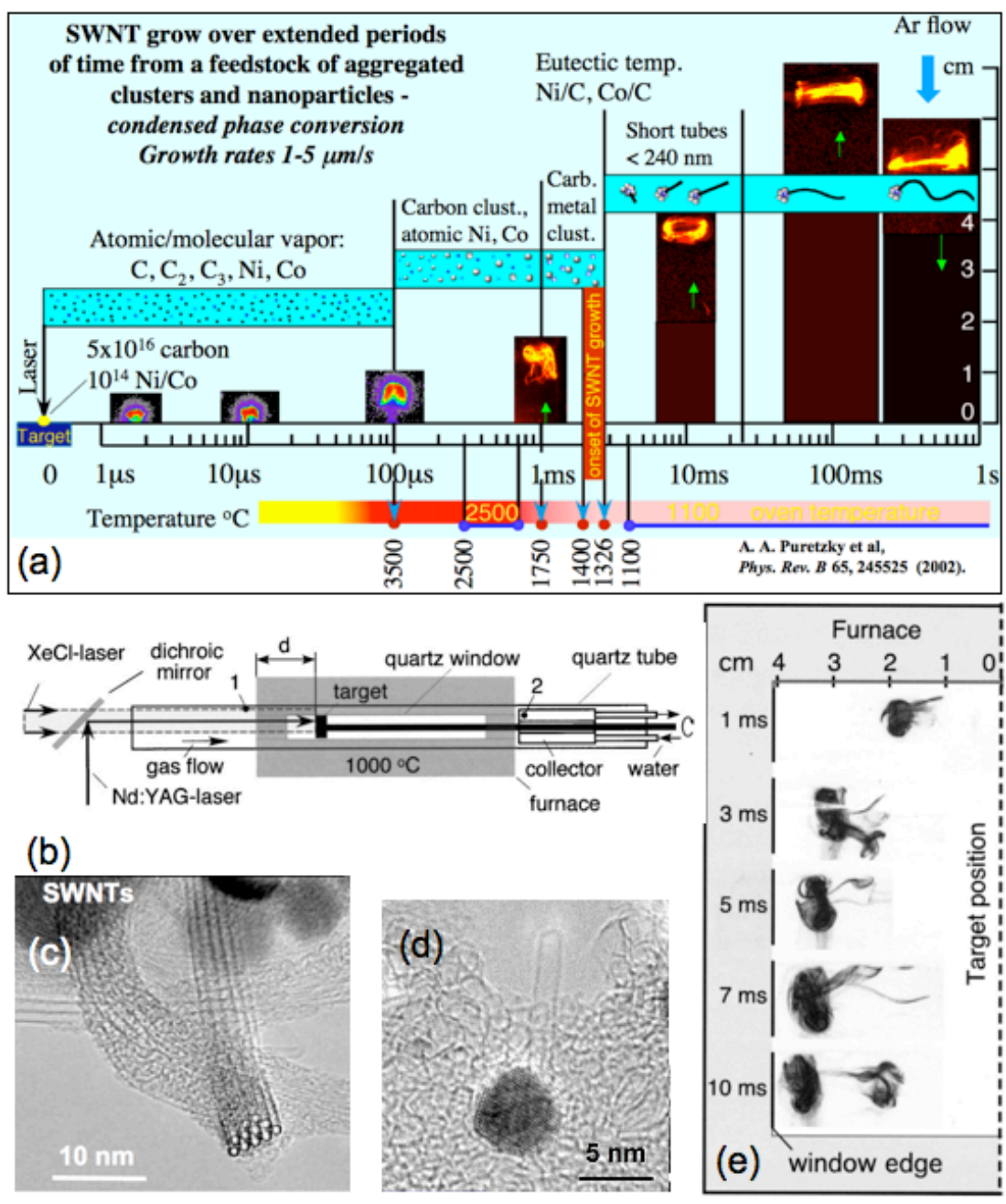

Fig. 2.6. (a) Summary of time-resolved imaging, spectroscopy, and temperature measurements of SWNT synthesis by laser vaporization. SWNT growth occurs at extended times from condensed carbon confined within a vortex ring at rates of 1-5 microns/second. (b) Schematic of the windowed laser oven used in the time-restricted growth experiments incorporating a second, time-delayed $\mathrm{XeCl}$ laser. (c) SWNT bundle typical of extended growth times (d) Short SWNT "seed" emanating from a $5 \mathrm{~nm} \mathrm{NiCo}$ nanoparticle resulting from time-restricted growth (e) Rayleigh scattering images of the plume formed within the windowed portion of the furnace, just prior to exiting the furnace for rapid quenching of the growth. After Ref. [27]

cumulative regime of laser ablation is very useful for synthesis of SWNTs and other nanomaterials when long-term confinement of the ablated material is required. 


\section{Laser Diagnostics and Controlled Chemical Vapor Deposition of Carbon Nanotubes}

As described in Figure 2.7, laser-based diagnostics have also recently been applied to understand and control the growth of carbon nanotubes by chemical vapor deposition (CVD), providing some of the first direct kinetics measurements and growth rates measured in situ. [28,29]
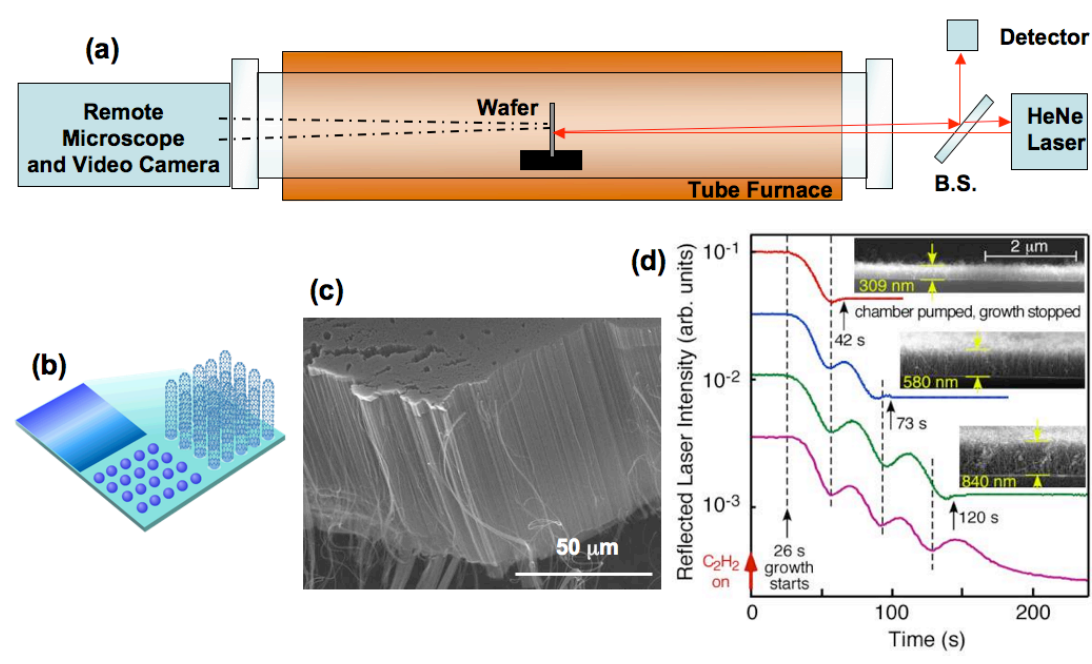

Fig. 2.7. (a) Schematic of apparatus used for in situ measurement of carbon nanotube growth kinetics. A CW-HeNe laser beam is reflected from a vertically-standing substrate through the end window of a tube furnace. A remote microscope and video camera may be used from the opposite window to record growth to millimeters lengths. (b) Schematic of chemical vapor deposition (CVD) growth of vertically-aligned nanotube arrays (VANTAs). A thin film catalyst is deposited (usually $10 \mathrm{~nm}$ of Al as a buffer layer on $\mathrm{Si}$, then $\sim 1 \mathrm{~nm}$ of Fe as catalyst, and sometimes $\sim 0.2 \mathrm{~nm}$ of Mo as a mixed catalyst). During heating in a tube furnace to $550-950^{\circ} \mathrm{C}$ in $\mathrm{Ar} / \mathrm{H}_{2}$ mixtures, the catalyst film roughens into nanoparticles. A mixture of hydrogen, argon and acetylene is then introduced (or another hydrocarbon such as methane, ethylene, etc.) and nanotubes nucleate and grow from the metal catalyst nanoparticles to form dense, selfaligned arrays. (c) SEM micrograph of a cleaved VANTA. The Si wafer is at the bottom, and the top of the array indicates the porous nature of the block of continuous nanotubes. Most VANTAs are $<10$ vol.\% dense. (d) As the nanotubes begin to grow, the HeNe laser beam is reflected from both the metallized Si substrate and the top of the growing nanotube array, resulting in Fabry-Perot interference fringes measured at the detector (in addition to signal attenuation due to absorption). Each fringe corresponds to $\sim 300 \mathrm{~nm}$ of array height. The growth rate of the nanotubes can be directly measured in situ, and the length of the nanotube arrays can be controlled. After Refs. [23,24] 

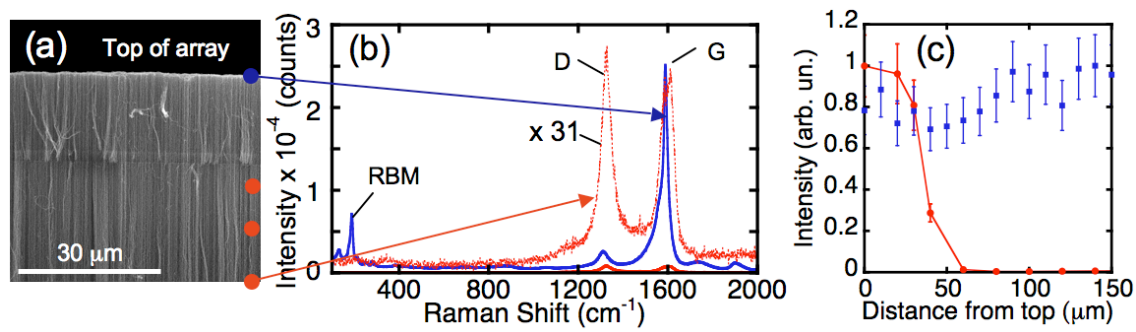

Fig. 2.8 (a) SEM image of the top of a VANTA array grown with different partial pressures of acetylene at 760 Torr $750^{\circ} \mathrm{C}$ in $2500 \mathrm{sccm} \mathrm{Ar} / \mathrm{H}_{2}$ gas mixture. Since the nanotubes grow from catalyst anchored at the substrate, the top of the array (grown with 1 sccm $\mathrm{C}_{2} \mathrm{H}_{2}$ ) reflects nanotubes which grew first, and display a high SWNT fraction displaying (b) Raman spectra $\left(\lambda_{\mathrm{ex}}=633 \mathrm{~nm}\right)$ with pronounced RBM modes and a high G/D Raman band ratio (blue curve). The number of walls in the array can be adjusted, in accordance with the growth model, by an oversupply in feedstock. Thus, the bottom part of the array (grown with $10 \mathrm{sccm} \mathrm{C}_{2} \mathrm{H}_{2}$ ) displays a lack of SWNTs and a Raman spectrum reflecting MWNTs (red curves, actual intensity and scaled by a factor of 31). (c) Raman profiling of the array (laser polarization parallel to the nanotube alignment) shows a dropoff in RBM intensity (red circles and line) following the change to $10 \mathrm{sccm}$ feedstock supply after 15 microns of initial growth. An array grown at $1 \mathrm{sccm}$ constant supply (blue square points) is shown for comparison. [24]

Using the results from in situ growth rate measurements in which the temperature, gas flow, and hydrocarbon concentration were varied, a kinetics model was developed to fit the measured growth rates and terminal lengths of the VANTAs. Activation energies for the different processes were determined, and the optimal growth conditions to produce long nanotube arrays were predicted. ${ }^{18}$ By measuring the number of walls for the nanotubes grown under different conditions, it was possible to understand how the number of walls of a nanotube grown from a catalyst nanoparticle depends on the feedstock supply. The model predicts that for a particular catalyst the fastest growing nanotube is a single-wall carbon nanotube at a given temperature and feedstock supply, however with an oversupply of feedstock more nanotube walls are formed. [30] Typically, the number of walls found in continuously grown VANTAs changes with time, as revealed by Raman spectroscopy in Figure 2.8.

Lasers therefore permit in situ remote characterization of nanotube growth kinetics via time-resolved reflectivity. Moreover, through Raman spectroscopy, the presence and diameter of SWNTs can be assessed through the presence of the radial breathing modes (RBM's) in microRaman profiling of nanotubes grown under different conditions (Fig. 2.8(b)). Similarly, the number of defects in the nanotubes 
can be assessed by a comparison of the G:D Raman band ratio intensity (Fig. $2.8(\mathrm{c}))$.

However, laser irradiation can also be used to alter the activity of the metal catalysts used for nanotube growth. Through KrF-laser processing of multilayer metal catalyst films prior to CVD, remarkable changes in subsequent VANTA growth rates, terminal heights, nanotube diameters and wall numbers were observed. [30] Depending upon the fluence, growth was either stunted or enhanced, however in the case of Figure 2.9(a) the laser-processed regions resulted in over three times the growth rate and terminal length of the unprocessed regions, resulting in $1.4 \mathrm{~cm}$ tall nanotube pillars. HRTEM analysis of the nanotubes in the tall pillars and shorter mats revealed a much narrower distribution of nanotube diameters and wall
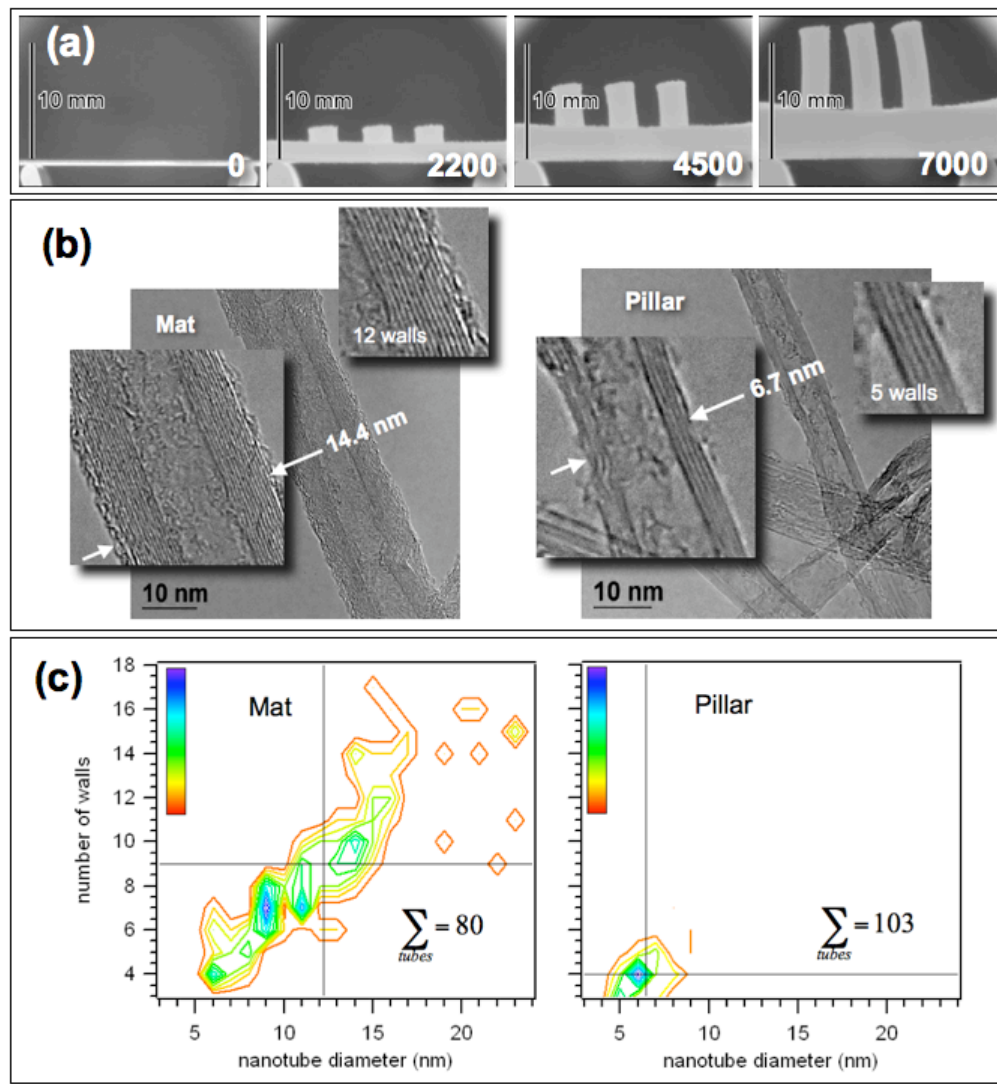

Figure 2.9 (a) Time lapse images of VANTA growth on unprocessed and laser-irradiated $\mathrm{Fe}(1 \mathrm{~nm}) / \mathrm{Mo}(0.2 \mathrm{~nm})$ films on $10 \mathrm{~nm}$ Al-coated Si wafers, at the indicated time in seconds. (b) HRTEM images reveal that the taller pillars of nanotubes in the laser processed areas have ewer walls and are narrower in diameter than those in the mat (unirradiated area). (c) Distributions of nanotube wall number vs. nanotube diameter shows that the laser processed areas in the pillars have greatly reduced diameter distributions and smaller diameters. (Reproduced with permission from [30]). 
numbers in the laser-processed regions, corresponding to slimmer, faster-growing nanotubes. Despite their narrow diameter, the laser-processed regions were more densely packed, and weight measurements showed that on a per unit substrate area basis the processed regions were far more catalytically active than the unprocessed area. Thus, laser processing appears highly promising to influence and control the catalytic activity of metal alloy films used for CVD.

Lasers can also be used to provide unique growth conditions for CVD. Recently, we utilized infrared laser pulses to provide well-defined growth periods for carbon

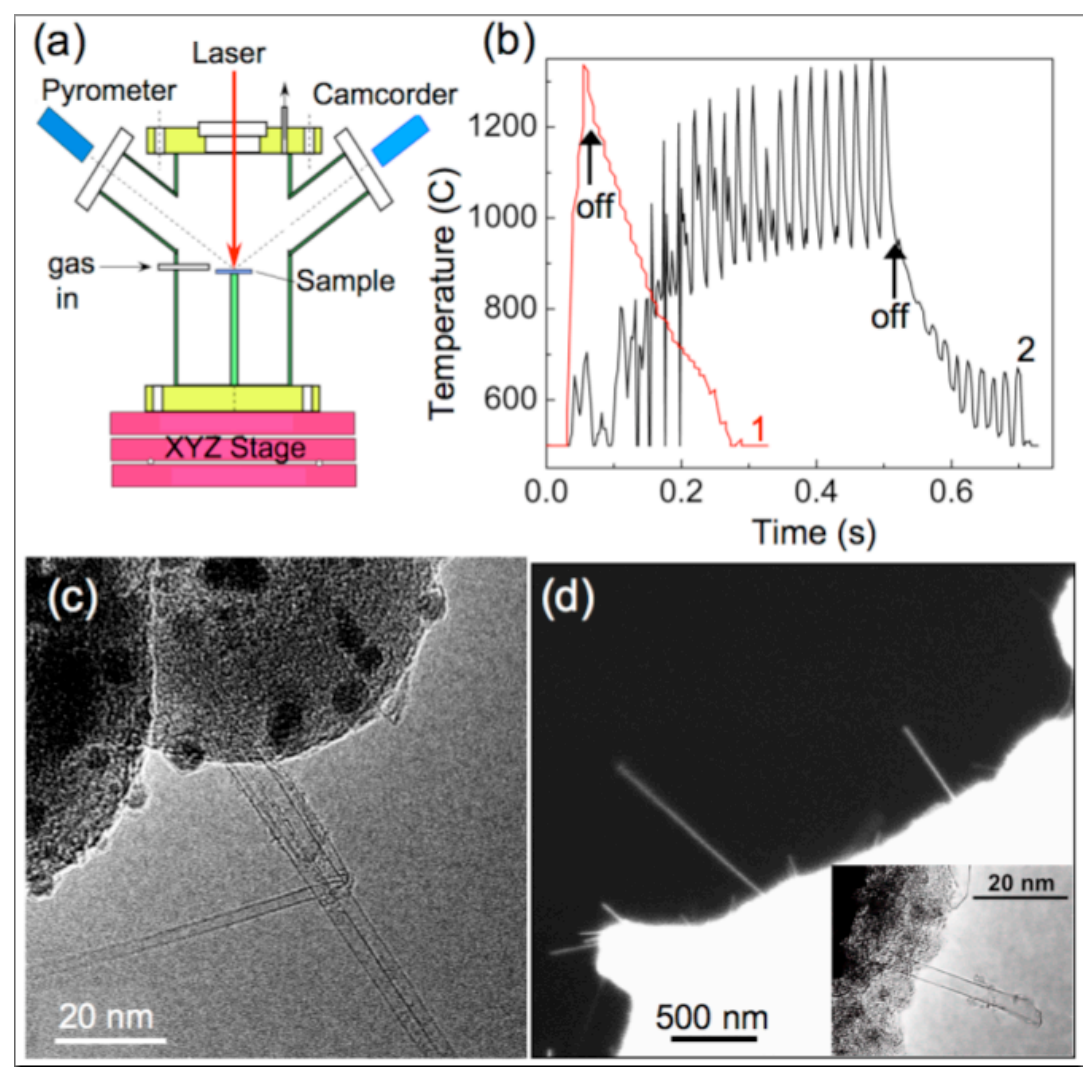

Figure 2.10 (a) Schematic of PLA-CVD vacuum chamber. (b) Time-dependent temperature profile of a $1 \mathrm{~cm}^{2} \mathrm{Si} / \mathrm{SiO}_{2}$ wafer by a single $50 \mathrm{~ms}$ laser pulse (1), and a Mo TEM grid from 25 pulses of $5 \mathrm{~ms}$ width (2). Arrows show the time when laser irradiation is terminated. (c), TEM image of CNTs grown on a Mo grid coated with $1 \mathrm{~nm}$ $\mathrm{Fe} / \mathrm{Al}_{2} \mathrm{O}_{3}$ by 1500 laser pulses. (d) SEM of nanotubes grown using 20 pulses on an identically prepared grid as (c). Inset shows a TEM image of the end of a nanotube free of catalyst particle. [31] 
nanotubes on Si wafers and TEM grids. As shown in Figure 10, laser heating of the substrates within a CVD chamber was monitored in situ by fast optical pyrometry. The study found that exclusively single-wall carbon nanotubes form by rapid laser heating, and at the highest recorded rates of 100 microns/sec. Interestingly, growth was found not to occur incrementally on successive laser pulses, that is, once the catalyst particle was cycled it was catalytically inactive. Nevertheless, on successive laser pulses new catalyst particles may nucleate and grow a nanotube. This feature was used to demonstrate the direct-writing of SWNT field-effect transistors on prepatterned electrodes decorated with catalyst. [31]

\section{Summary}

In summary, a variety of laser interactions for nanomaterial synthesis have been described. Laser vaporization is a powerful exploratory tool in nanomaterial synthesis, providing congruent and complete vaporization of solids to permit the clean self-assembly of nanomaterials in gases, without interactions with substrates. Fullerenes, photoluminescent silicon quantum dots, and single-wall carbon nanohorns are all examples of novel nanomaterials discovered by laser vaporization in different fluence ranges and background gas pressures.

Lasers are also used in the catalyst-assisted growth of nanomaterials, such as carbon nanotubes. Here, carbon nanotube growth by both laser vaporization and CVD were described. In both cases, laser interactions are used to remotely characterize the size, composition, and electronic structure of intermediate species and track their dynamics, as well as remotely provide some of the first growth kinetics information of the nanotubes as they grow. During laser vaporization, laser induced fluorescence and Rayleigh scattering utilizing time-delayed probe laser pulses were used to understand the timescales for growth. During CVD, time-resolved laser reflectivity from the growing nanotube arrays provides both length and density information of the arrays, and in situ Raman spectroscopy of SWNTs can be used to understand diameters and defects.

Lasers are emerging as instruments to modify and control growth,, for example in the described laser processing of catalysts used for CVD to alter their activity, and also in the pulsed heating of metal catalysts to nucleate and grow discrete SWNTs in precise locations. A great variety of other effects remain to be described and explored, however it is certain that lasers - with their remote delivery of energy will be used to alter the synthesis conditions and characterize the effects for the growth of novel nanomaterials of the future. 


\section{7 - Acknowledgments}

The authors gratefully acknowledge the technical assistance of Pam Fleming and technical discussions with D.H. Lowndes, R.F. Wood, S. Pannala, and J. Wells. Fundamental measurements of nanotube and nanohorn growth [AA, DG, CR, GE] funded by DOE-BES Division of Materials Science. Characterization measurements, including research on rapid laser heating for device functionality with Dongning Yuan and Jie Liu from Duke University, performed through user projects at the Center for Nanophase Materials Sciences [ZL, DSB, II, KX] which is supported by the U. S. Dept. of Energy Basic Energy Sciences, Division of Scientific User Facilities.

\section{References}

1. T. G. Dietz, M. A. Duncan, D. E. Powers, and R. E. Smalley, J. Chem. Phys. 74, 6511 (1981).

2. H. W. Kroto, J. R. Heath, S. C. O’Brien, R. F. Curl, and R. E. Smalley, Nature 318, 162 (1985).

3. A. Inam, X. D. Wu, T. Venkatesan, S. B. Ogale, C. C. Chang, and D. Dijkkamp, Appl. Phys. Lett. 51, 619 (1987).

4. D. B. Geohegan, Ch.4 in D. B. Chrisey and G. K. Hubler (editors), Pulsed Laser Deposition of Thin Films (Wiley, New York, 1994).

5. A. Thess, R. Lee, P. Nikolaev, H. Dai, P. Petit, J. Robert, C. Xu, Y. H. Lee, S. G. Kim, A. G. Rinzler, D. T. Colbert, G. E. Scuseria, D. Tomanek, J. E. Fischer, and R. E. Smalley, Science 273(5274), 483-487 (1996).

6. A. M. Morales and C. M. Lieber, Science 279(5348), 208-211 (1998).

7. X. Duan and C. M. Lieber, Adv. Mater. 12(4) 298-302 (2000).

8. D. H. Lowndes, D. B. Geohegan, A. A. Puretzky, D. P. Norton, and C. M. Rouleau, Science 273 (5277), 898-903 (1996).

9. A. A. Puretzky, D. B. Geohegan, G. E. Jellison, and M. M. McGibbon, Applied Surface Science 96-8, 859-865 (1996).

10. A. A. Puretzky, D. B. Geohegan, X. Fan, and S. J. Pennycook, Appl. Phys. Lett. 76, 182-184 (2000). 
11. D. B. Geohegan, A. A. Puretzky, and D. J. Rader, Applied Physics Letters $\mathbf{7 4}$ (25), 3788-3790 (1999).

12. R. F. Wood, K. R. Chen, J. N. Leboeuf, A. A. Puretzky, and D. B. Geohegan, Physical Review Letters 79 (8), 1571-1574 (1997).

13. R. F. Wood, J. N. Leboeuf, D. B. Geohegan, A. A. Puretzky, and K. R. Chen, Physical Review B 58 (3), 1533-1543 (1998).

14. H. Shinohara, Rep. Prog. Phys. 63, 843-892 (2000).

15. C. N. R. Rao, G. U. Kulkarni, P. J. Thomas, Springer Series in Materials Science 95, ed. by C. N. R. Rao, G. U. Kulkarni, P. J. Thomas. Ch. 2., 2007.

16. Zel'dovich and Raizer, Physics of Shock Waves and High-Temperature Hydrodynamic Phenomena, Vol.2, (Academic Press) ISBN: $012778702 X$ (1967)

17. D. B. Geohegan, A. A. Puretzky, G. Duscher, and S. J. Pennycook, Applied Physics Letters 72 (23), 2987-2989 (1998) and Applied Physics Letters 73 (4), 438-440 (1998).

18. R. E. SMALLEY, Acc. Chem. Res. 25, 98-105 1992.

19. D. B. Geohegan, A. A. Puretzky, R. L. Hettich, X.-Y. Zheng, R. E. Haufler, and R. N. Compton, Trans. Mat. Res. Soc. Journal, 17, 349 (1994).

20. S. Iijima, M. Yudasaka, R. Yamada, S. Bandow, K. Suenaga, F. Kokai, and K.Takahashi, Chem. Phys. Lett. 309, 165-170 (1999).

21. P.J.F. Harris, S.C. Tsang, J.B. Claridge, S.C. Sang, J.B. Claridge, M.L. Green, Journal of the Chemical Society-Faraday Transactions, 90, pp 2799 (1994).

22. D. Kasuya, M. Yudasaka, K. Takahashi, F. Kokai, and S. Iijima, J. Phys. Chem. B 106, 4947-4951 (2002).

23. A.A. Puretzky, D.J. Styers-Barnett, C.M. Rouleau, H. Hu, B. Zhao, I.N. Ivanov, and D.B. Geohegan, Appl. Phys. A, (In Press).

24. D. B. Geohegan, A. A. Puretzky, D. Styers-Barnett, H. Hu, B. Zhao, H. Cui, C. M. Rouleau, G. Eres, J. J. Jackson, R. F. Wood, S. Pannala, and J. C. Wells, Physica Status Solidi B-Basic Solid State Physics 244 (11), 39443949 (2007). 
25. A. Thess, R. Lee, P. Nikolaev, H. Dai, P. Petit, J. Robert, C. Xu, Y. H. Lee, S. G. Kim, A. G. Rinzler, D. T. Colbert, G. E. Scuseria, D. Tomanek, J. E. Fischer, and R. E. Smalley, Science 273(5274), 483-487 (1996).

26. A. A. Puretzky, D. B. Geohegan, X. Fan, and S. J. Pennycook, Appl. Phys. A 70, 153-160 (2000).

27. A. A. Puretzky, H. Schittenhelm, X. Fan, M. J. Lance, L. F. Allard, and D. B. Geohegan, Phys. Rev. B, 65, 245425/1 (2002).

28. D. B. Geohegan, A. A. Puretzky, I. N. Ivanov, S. Jesse, G. Eres, and J. Y. Howe, Appl. Phys. Lett. 83(9), 1851-1853 (2003).

29. A. A. Puretzky, D. B. Geohegan, S. Jesse, I. N. Ivanov, and G. Eres, Appl. Phys. A, Mater. Sci. Process. 81(2), 223-240 (2005).

30. R. F. Wood, S. Pannala, J. C. Wells, A. A. Puretzky, and D. B. Geohegan, Physical Review B 75 (23) (2007).

31. C. M. Rouleau, G. Eres, H. Cui, H.M. Christen, A..A. Puretzky, and D.B. Geohegan, Appl. Phys. A (In Press).

32. Z. Liu, D.J.Styers-Barnett, A.A.Puretzky, C.M. Rouleau, D. Yuan, I. N. Ivanov, K.Xiao, J.Liu, D. B. Geohegan, Appl. Phys. A (In Press) published online DOI 10.1007/s00339-008-4804-8 (2008).. 